\title{
A PERIOD MAPPING IN UNIVERSAL TEICHMÜLLER SPACE
}

\author{
SUBHASHIS NAG
}

\begin{abstract}
In previous work it had been shown that the remarkable homogeneous space $M=\operatorname{Diff}\left(S^{1}\right) / \operatorname{PSL}(2, \mathbb{R})$ sits as a complex analytic and Kähler submanifold of the Universal Teichmüller Space. There is a natural immersion $\Pi$ of $M$ into the infinite-dimensional version (due to Segal) of the Siegel space of period matrices. That map $\Pi$ is proved to be injective, equivariant, holomorphic, and Kähler-isometric (with respect to the canonical metrics). Regarding a period mapping as a map describing the variation of complex structure, we explain why $\Pi$ is an infinite-dimensional period mapping.
\end{abstract}

\section{INTRODUCTION}

Let $\operatorname{Diff}\left(S^{1}\right)$ denote, as usual, the infinite-dimensional Lie group of orientation preserving $C^{\infty}$ diffeomorphisms of the unit circle. In a previous paper [12] we showed that the canonical complex analytic structure and Kähler metric, $g$, that exist on the homogeneous space $M=\operatorname{Diff}\left(S^{1}\right) / \operatorname{Möb}\left(S^{1}\right)$ coincide exactly with the canonical Ahlfors-Bers complex structure and the (generalised) Weil-Petersson Kähler structure of the Universal Teichmüller Space $T(1)$. This identification was via the natural injection (I) of $M$ into $T(1)$, where $T(1)$ is thought of as the homogeneous space of all quasi-symmetric homeomorphisms of $S^{1}$ modulo the Möbius subgroup.

In this paper we study another natural embedding $(\Pi)$ of $M$ into the infinitedimensional version, $D_{\infty}$, of the Siegel disc (or generalised upper halfspace) of period matrices. These matrices (suitable symmetric Hilbert-Schmidt operators) comprising $D_{\infty}$ are symmetric with complex entries, so $D_{\infty}$ is a complex manifold (infinite-dimensional bounded domain). As in finite dimensions, so also here the Siegel symplectic (Kähler) metric, $h$, exists on this homogeneous manifold. One main purpose here is to announce that, like $I$, the map $\Pi$ is completely natural (in that it respects all the structures); namely, $\Pi$ is an equivariant, holomorphic, and Kähler isometric immersion of $M$ into $D_{\infty}$. Thus, combining this with the results proved by this author in [12, Part II], one can assert that on the complex submanifold $M$ of the Teichmüller space, the WeilPetersson metric coincides with the Siegel symplectic metric.

Considering $M$ as the submanifold of "smooth" complex structures in the Teichmüller space, we can interpret the mapping $\Pi$ as a period mapping that associates to each point of $M$ its corresponding matrix of periods. The idea is to look at the period map in P. Griffiths's way as a map describing the variation of Hodge and complex structure. We will provide an exposition.

Received by the editors May 21, 1991 and, in revised form, August 20, 1991.

1991 Mathematics Subject Classification. Primary 32G15, 32G20, 30F10, 30F60, 81T30.

This paper was presented as an invited lecture on May 27, 1991, at the second International Symposium on Topological and Geometrical Methods in Field Theory, Turku, Finland, (May 27June 1, 1991). Sponsored by Academy of Finland et al. 
As explained in [12], that work was intimately related to and motivated by string physics. Once again, physicists have been looking at the map $\Pi$ and have made several claims. The various infinite-dimensional spaces appearing in the set-up are all somehow universal moduli spaces and may turn out to be important in a nonperturbative formulation of string theory. See Nag [10].

\section{THE MAP $\Pi$}

The method of defining $\Pi$ stems from a faithful representation of $\operatorname{Diff}\left(S^{1}\right)$ in the infinite-dimensional symplectic group, say $\mathrm{Sp}$, that was introduced by G. Segal $[13, \S 5]$. (Segal's motivation is to obtain the metaplectic representation of $\mathrm{Sp}$, but that need not concern us here.) Recall that if a (real) vector space $V$ is equipped with a skew-symmetric, nondegenerate bilinear form, $S$, then the linear automorphisms $T \in \mathrm{GL}(V)$ that preserve the form $S$ (viz., $S\left(T v_{1}, T v_{2}\right)=S\left(v_{1}, v_{2}\right)$ for all $v_{1}, v_{2}$ in $\left.V\right)$ constitute the symplectic subgroup $\mathrm{Sp}(V, S) \subset \mathrm{GL}(V)$.

Consider the vector space

$$
V=C^{\infty} \operatorname{Maps}\left(S^{1}, \mathbb{R}\right) / \mathbb{R} \text { (constant maps). }
$$

The quotienting by the one-dimensional subspace of constant maps means that one is normalizing the 0 -th Fourier coefficient to vanish. $V$ is equipped with a natural nondegenerate skew-symmetric bilinear form $S: V \times V \rightarrow \mathbb{R}$ (Segal [13]), given by

$$
S(\sigma, \tau)=\int_{S^{1}} \sigma(\theta)(d \tau / d \theta) d \theta
$$

Then $\operatorname{Diff}\left(S^{1}\right)$ acts on $V$, by the obvious precomposition, preserving this bilinear form $S$. Hence the diffeomorphism group becomes a subgroup of the real symplectic automorphisms of $(V, S)$. Actually one deals with a restricted subgroup of the symplectic automorphisms, denoted $\mathrm{Sp}_{0}(V)$, which already contains $\operatorname{Diff}\left(S^{1}\right)$ (see [13]). (This is an analytic detail to simplify the infinitedimensional considerations, since $\operatorname{Sp}_{0}(V)$ elements have a natural norm.)

To proceed further we recall the definition of a positive polarization of the space $V$ with respect to $S$. Let $V_{\mathbb{C}}$ denote the complexification of $V$. Then a decomposition $V_{\mathbb{C}}=W \oplus \bar{W}$ such that (the complexification of) $S$ takes zero values on arbitrary pairs from $W$ is called a polarization and $W$ is called a (maximal) isotropic subspace for $S$. The assignment

$$
\left\langle w_{1}, w_{2}\right\rangle=i S\left(\bar{w}_{1}, w_{2}\right)
$$

is a Hermitian inner product on $W$, and the decomposition is a positive polarization if this is positive definite. In that case $W$, its conjugate, and hence $V_{\mathbb{C}}$ itself, can be completed to Hilbert spaces with respect to this Hermitian inner product. We will henceforth identify a positive polarization with the isotropic subspace $W$ determining it. Here is the fiducial positive polarization for $(V, S)$ : take $W=W_{+}$to be the subspace of $V_{\mathbb{C}}=C^{\infty} \operatorname{Maps}\left(S^{1}, \mathbb{C}\right) / \mathbb{C}$ consisting of those mappings having only positive index Fourier components. Note that $\bar{W}_{+}=W_{-}$, and also the fundamental fact that the image under (the $\mathbb{C}$-linear extension of ) a symplectic automorphism of a positive isotropic subspace is again such a subspace. In fact, $\operatorname{Sp}(V, S)$ acts transitively on the space 
of all positive polarizations, and the stabilizer subgroup at $W$ is evidently identifiable with the unitary group $U(W,\langle\rangle$,$) . It follows that the homogeneous$ space $\mathrm{Sp} / U$ can be identified with the family of positive polarizations of $V$. Passing to the restricted subgroup $\mathrm{Sp}_{0}$ one finds that $\mathrm{Sp}_{0} / U$ represents a class of ("bounded") positive polarizations of $V$, say $\operatorname{Pol}_{0}(V)$, and either of these spaces can be easily identified with the Siegel disc of infinite dimension (Segal $[13$, p. 316]),

$$
\begin{aligned}
D_{\infty}= & \left\{\text { All Hilbert-Schmidt complex linear operators } Z: W_{+} \rightarrow W_{-}\right. \\
& \text {such that } Z \text { is symmetric (w.r.t. } S) \text { and } I-Z \bar{Z} \text { is positive definite }\} .
\end{aligned}
$$

For instance, the identification between $D_{\infty}$ and $\operatorname{Pol}_{0}(V)$ is by associating to $Z \in D_{\infty}$ the positive isotropic subspace $W$ that is the graph of the operator $Z$. (The origin in $D_{\infty}$ corresponds to the fiducial polarization $W_{+}$.) In finite dimension (genus $g$ Riemann surfaces) this identification corresponds to taking $W$ to be the row span of the "full period matrix" $(Z, I)$; then $W$ is a positive isotropic $g$-dimensional complex subspace of $\mathbb{C}^{2 g}$ (equipped with standard skew form). The current set-up thus agrees well with the classical case, and we have in infinite dimension the Siegel disc appearing in the various incarnations

$$
D_{\infty}=\operatorname{Sp}_{0} / U=\operatorname{Pol}_{0}(V) \hookrightarrow \operatorname{Gr}\left(W_{+}, V_{\mathbb{C}}\right) .
$$

Here the subspaces $W \in \operatorname{Pol}_{0}(V)$ comprise a complex analytic submanifold of the complex Grassmannian of subspaces of $V_{\mathbb{C}}$ that are graphs of (not necessarily symmetric) linear operators of Hilbert-Schmidt type from $W_{+}$to $W_{-}$. This complex structure on $\operatorname{Pol}_{0}(V)$ (identified as explained with $D_{\infty}$ ) is easily checked to be the same as the complex structure of $D_{\infty}$ as a family of (symmetric with respect to $S$ ) complex operators.

We are finally ready to define $\Pi$.

Lemma. In Segal's representation $\operatorname{Diff}\left(S^{1}\right) \rightarrow \mathrm{Sp}_{0}(V)$, the diffeomorphisms that map into the unitary subgroup $U$ of $\mathrm{Sp}_{0}$ comprise precisely the 3-parameter subgroup of Möbius transformations on $S^{1}$. Consequently one obtains an injective equivariant mapping

$$
\Pi: \operatorname{Diff}\left(S^{1}\right) / \operatorname{Möb}\left(S^{1}\right) \rightarrow \operatorname{Sp}_{0} / U=D_{\infty} .
$$

The proof boils down to showing that a holomorphic self-map of the disc that extends continuously to the boundary as a diffeomorphism of $S^{1}$ must be a Möbius transformation. This follows, for example, by the argument principle. That $\Pi$ is equivariant (with respect to corresponding right translations on domain and target) is easily verified.

\section{HOLOMORPHY OF $\Pi$}

Theorem. $\Pi$ is a holomorphic immersion.

The method is to compute the derivative of $\Pi$ at the origin of $M$ and show that it is a complex linear map of the tangent space of $M$ to the tangent space of the Grassmannian above. By equivariance it is enough to verify this at the origin.

Let me first explain the canonical complex structure on the homogeneous space $M$. The Lie algebra of the Lie group $\operatorname{Diff}\left(S^{1}\right)$ is the algebra of smooth 
$\left(C^{\infty}\right)$ real vector fields on $S^{1}$. The tangent space at the origin (i.e., coset of the identity) of $M$ corresponds to those smooth vector fields $v=u(\theta) \partial / \partial \theta$ such that in the Fourier expansion

$$
u(\theta)=\sum_{m=-\infty}^{\infty} u_{m} e^{i m \theta}
$$

one has $u_{-1}=u_{0}=u_{1}=0$. Note that reality of $v$ implies $\overline{u_{m}}=u_{-m}$. One easily sees that the three middle Fourier coefficients vanish because an infinitesimal Möbius transformation allows one to normalize precisely these three coefficients.

The canonical homogeneous almost-complex structure $J$ on $M$ is now defined simply by "conjugation of Fourier Series," namely,

$$
J\left(u(\theta) \frac{\partial}{\partial \theta}\right)=u^{*}(\theta) \frac{\partial}{\partial \theta},
$$

where

$$
u^{*}(\theta)=-\sum_{m=-\infty}^{\infty} i \operatorname{sgn}(m) u_{m} e^{i m \theta} .
$$

This defines $J$ at the origin on the homogeneous Fréchet manifold $M$; elsewhere $J$ is transported by right translations. It is easy to check (at least at the formal level), that this almost complex structure on $M$ is integrable. Thus $M$ is canonically a homogeneous complex manifold. The complex structure above arises naturally from various points of view, for instance, from the KirillovKostant coadjoint orbit theory and also in work of Pressley et al. See references $[1-4,16,17]$.

Now, the tangent space to the Grassmannian at a point $H$ can be canonically identified with the linear space $\operatorname{Hom}\left(H, V_{\mathbb{C}} / H\right)$ (only those homomorphisms satisfying a Hilbert-Schmidt boundedness condition are included). We now exhibit the derivative of $\Pi$ from the tangent space of $M$ to the tangent space of the Grassmannian.

Proposition. The derivative of $\Pi$ at the origin of $M$ carries the vector field $v=u(\theta) \partial / \partial \theta$ to the following homomorphism $\eta_{v} \in \operatorname{Hom}\left(W_{+}, W_{-}\right)$:

$$
\eta_{v}(\sigma)=\pi_{-}\left(u(\theta) \sigma^{\prime}(\theta)\right) \text { for any } \sigma \in W_{+},
$$

where $\pi_{-}$denotes the projection of $V_{\mathbb{C}}$ onto $W_{-}$and prime denotes differentiation with respect to $\theta$.

The proof of (10) is straightforward after one has properly identified the various spaces involved.

To establish the holomorphy of $\Pi$ one needs to prove that the homomorphism $\eta_{J v}$ is $i$ times $\eta_{v}$. Recall that the negative index Fourier coefficients of $J v$ are just the corresponding coefficients of $v$ multiplied by $i$. On the other hand, any $\sigma$ from $W_{+}$(therefore any $\sigma^{\prime}$ ) has only positive index Fourier components. Application of formula (10), therefore, gives what we wish.

\section{3. $\Pi$ IS AN ISOMETRY}

There is a unique homogeneous Kähler metric, $g$, available on $M$. In Part II of [12] I had proved that this metric is precisely the Weil-Petersson metric on the Teichmüller spaces. 
Here is one way to explain how $g$ arises. Since $M$ is a coadjoint orbit in the coadjoint representation of the Virasoro group, (see Witten [16]), one has, as always, the natural Kirillov-Kostant symplectic form on these homogeneous manifolds. This symplectic form is compatible with the complex structure seen above, and we thus get the Kähler structure $g$ on $M$. In a less sophisticated fashion, it is actually easy to see that $g$ is the unique homogeneous Kähler metric (up to a numerical scaling parameter) possible on $M$. The number 26, namely, the dimension of bosonic spacetime, appears entrenched in the curvature of $g$. See calculations of Bowick, Rajeev, Lahiri, and Zumino in the references. Explicitly, this canonical $g$, at the origin of $M$ has the Hermitian pairing (see [12, Part II])

$$
g(v, w)=\left[\sum_{m=2}^{\infty} v_{m} \overline{w_{m}}\left(m^{3}-m\right)\right] .
$$

Here $v$ and $w$ are two smooth real vector fields on $S^{1}$ representing tangent vectors to $M$ (see equation (7)), and the $v_{m}, w_{m}$ are the respective Fourier coefficients. Elsewhere on $M, g$ is of course transported by right translations isometries.

The Siegel symplectic metric, $h$, (note Siegel [15]) exists on each finitedimensional Siegel disc $D_{g}$ as the unique Kähler metric on that bounded domain for which the full holomorphic automorphism group $\operatorname{Sp}(2 g, \mathbb{R})$ acts as isometries. It generalises without trouble to Segal's infinite-dimensional version $D_{\infty}$ explained above. The pairing given by $h$ on the symmetric elements of $\operatorname{Hom}\left(W_{+}, W_{-}\right)$, i.e., on the tangent space at the origin of $D_{\infty}$, turns out, after some work, to be

$$
h(\phi, \psi)=\text { trace of } \phi \circ \bar{\psi},
$$

where the conjugate of $\psi$ maps $W_{-}$to $W_{+}$, so taking trace makes sense (and gives a finite answer).

Theorem. $\Pi$ is an isometric immersion of $(M, g)$ into $\left(D_{\infty}, h\right)$.

Again utilising equivariance and the fact that $g$ and $h$ are homogeneous metrics we only need check at the origin. One calculates the trace of $\eta_{v}$ composed with conjugate of $\eta_{w}$ and finds interestingly that the answer is as predicted by formula (11). The computation is finally a finite-series summation. See [10] for details.

\section{Dependence of $\Pi$ on Beltrami coefficients}

Here is the explicit form of the period matrix in $D_{\infty}$ associated to a given Beltrami coefficient $\mu$ (representing a Teichmüller point $[\mu]$ ). The symplectic automorphism $T_{\mu}$ of $V$ arising from $\mu$ is $h \mapsto h \circ w_{\mu}, h \in V$. Here $w_{\mu}$ is the $\mu$-conformal self-homeomorphism of the unit disc $\Delta$, and we are using its boundary homeomorphism. The matrix for $T_{\mu}$ (complexified) in the standard orthonormal bases $\left\{e_{k}=e^{i k \theta} / \sqrt{|k|}\right\}$ for $V_{\mathbb{C}}=W_{+} \oplus W_{-}$has the form

$$
T_{\mu}=\left[\begin{array}{ll}
\frac{A}{B} & \frac{B}{A}
\end{array}\right] .
$$

Here $A: W_{+} \rightarrow W_{+}, B: W_{-} \rightarrow W_{+}, A=\left(\left(a_{p q}\right)\right), B=\left(\left(b_{p q}\right)\right)$. 
We obtain the fundamental formulas

$$
\begin{array}{rlrl}
a_{p q} & =\frac{1}{2 \pi} \frac{\sqrt{p}}{\sqrt{q}} \int_{0}^{2 \pi}\left(w_{\mu}\left(e^{i \theta}\right)\right)^{q} e^{-i p \theta} d \theta, & & p, q>0 \\
b_{r s} & =\frac{1}{2 \pi} \frac{\sqrt{r}}{\sqrt{s}} \int_{0}^{2 \pi}\left(w_{\mu}\left(e^{i \theta}\right)\right)^{-s} e^{-i r \theta} d \theta, & r, s>0 .
\end{array}
$$

Recall that the symplectic group acts by holomorphic automorphisms on $D_{\infty}: Z \mapsto(\bar{A} \circ Z+\bar{B}) \circ(B \circ Z+A)^{-1}$ where the symplectic matrix " $T$ " is in the block form (13). The zero matrix in $D_{\infty}$ corresponds to $\mu=0$, and we see that

$$
\Pi([\mu])=\bar{B} \circ A^{-1}
$$

with $A$ and $B$ given as above.

First variation of the period matrix. That the matrix (16) varies holomorphically with $\mu$ is already nontrivial (remember that $w_{\mu}$ varies only real analytically with $\mu$ ). Since we know the perturbation formula for $w_{\mu}$, we can actually write down the infinitesimal variation of the matrix entries of $\Pi(\mu)$ as $\mu$ varies.

Proposition. For $\mu \in L_{\infty}(\Delta)$ one has, as $t \rightarrow 0$,

$$
\Pi([t \mu])_{r s}=t \cdot \pi^{-1} \cdot \sqrt{r s} \iint_{\Delta} \mu(z) z^{r+s-2} d x d y+O\left(t^{2}\right) .
$$

Remark. The proposition corresponds precisely to the well-known Rauch variational formulas for finite genus period mappings. In fact, the holomorphic 1 -forms on $\Delta$ are generated by $\phi_{r}=z^{r-1}, r=1,2, \ldots$; so (17) is in harmony with the Rauch formula " $\iint \mu \phi_{r} \phi_{s}$ " for the variation of the $r s$ entry. (See [11, Chapter 4].)

\section{5. $\Pi$ AS A PERIOD MAPPING}

The map $\Pi$ links up with the variation of Hodge structure following P. Griffiths $[5,6]$. The idea is to interpret the vector space $V$ as the "first cohomology (with real coefficients) of a surface of infinite genus." The skew form $S$ can then be recognised as the cup product map on this cohomology vector space.

Now, a "complex structure", that is a point from the universal Teichmüller space, will produce a decomposition of the complexification of $V$ into its $H^{1,0}$ and $H^{0,1}$ subspaces. The $H^{1,0}$ subspace is a positive isotropic subspace for the cup product skew form because the condition of being positive isotropic is an implementation of the bilinear relations of Riemann. The decomposition so obtained is indeed a positive polarization of $V$. For the base complex structure " 0 ", we take $H^{1,0}$ to be $W_{+}$. Thus $\Pi$ becomes realised as an association between the smooth complex structures and their corresponding $H^{1,0}$ subspaces, as is expected of a period mapping in Griffiths's set-up. That $\Pi$ is injective (as we saw) is then Torelli's Theorem in this context.

One can also prove that $\Pi$ keeps track of the varying space of holomorphic 1 -forms as the complex structure varies. That is precisely the characteristic of the classical period mappings. See [10] for this point of view.

The infinitesimal Schottky locus. It is clear that the image $\Pi(M)$ in $D_{\infty}$ is the analog of the Schottky locus of Jacobians in the Siegel disc $D_{g}$. Using the formula (10) for $d \Pi$ we get a pretty description of the tangent to the "Schottky locus" $\Pi(M)$ : 
Theorem. The tangent space to $D_{\infty}$ at the origin is canonically identifiable with the complex symmetric matrices $\left(\left(\lambda_{p q}\right)\right)(p, q=1,2,3, \ldots)$ satisfying the Hilbert-Schmidt condition $\sum_{p=1}^{\infty} \sum_{q=1}^{\infty}\left|\lambda_{p q}\right|^{2}<\infty$. The image of $d \Pi$ at the origin consists of those that are of the form

$$
\lambda_{p q}=i \sqrt{p q} a_{(p+q)},
$$

where the sequence $\left(a_{2}, a_{3}, a_{4}, \ldots\right)$ is an arbitrary complex sequence whose $n$th term goes to zero faster than $|n|^{-k}$ for any $k>0$.

Remark. In finite genus $g$, the $(3 g-3)$-dimensional Teichmüller space sits via $\pi_{g}$ in the $g(g+1) / 2$-dimensional $D_{g}$. Here also we see that the number of independent entries in a $D_{\infty}$ tangent vector is growing quadratically with the block size, whereas the ones arising from Teichmüller points are determined just by their first column, i.e., grows linearly with block size. In this aspect also $\Pi$ generalises the $\pi_{g}$.

Remark. Since $D_{\infty}$ sits within the infinite-dimensional Grassmannian, the theory (of the tau function) developed in [14] by Segal-Wilson suggests that it may be possible to describe the image of $\Pi$ by solutions of $K-P$ equations; namely, an infinite-dimensional form of the Novikov conjecture could be valid.

Remark. In Part II of [12] the present author had shown a form of Mostow rigidity that implied that the Teichmüller spaces $T_{g}$ of compact Riemann surfaces of genus $g$ sit within the universal $T(1)$ cutting transversely the submanifold $M$. That result disallows the possibility of relating the finite-dimensional period maps $\pi_{g}: T_{g} \rightarrow D_{g}$ to $\Pi$ by simple restriction of domains. Nevertheless, it was possible in Part II of [12] to relate the Weil-Petersson metric on $T_{g}$ to the canonical metric $g$ of $M$ by a regulation of improper integrals. Some similar analysis may relate $\pi_{g}$ to $\Pi$.

In any case the result of $\S 3$ raises the following interesting question: How is $\pi_{g}^{*}$ (Siegel symplectic metric) related to the Weil-Petersson metric on $T_{g}$ ? Certainly they do not coincide in low genus! A connection between the volume form induced on $T_{g}$ by the Siegel metric and volume forms arising from the complex geometry of $T_{g}$ was shown in Nag [9].

Hong-Rajeev [7] have considered the mapping $\Pi$ as a map from the space of univalent functions (Riemann maps) to $D_{\infty}$; they have made several claims but have offered no proofs. They appear to be thinking of points of $\mathrm{Sp}_{0} / U$ as complex structures on $V$ rather than as polarizations. It seems to us that this does not quite fit requirements. Also, if one wishes to look at $\Pi$ as a map on univalent functions, namely, as a map on domains bounded by smooth Jordan curves, then one is forced to use the conformal welding correspondence to relate to $\operatorname{Diff}\left(S^{1}\right) / \operatorname{Möb}\left(S^{1}\right)$. (See [12, Part II] and Katznelson-Nag-Sullivan [8]). Using the welding it is possible for us to write formulas for $\Pi$ as a map on coefficients of Riemann maps (which are essentially nothing but Bers embedding holomorphic coordinates); however, we are unable to see how the formulas presented by Hong and Rajeev can be valid.

More details on this work will appear in [10] and elsewhere. 


\section{REFERENCES}

1. M. Bowick, The geometry of string theory, 8th Workshop on Grand Unification, Syracuse, NY 1987.

2. M. Bowick and A. Lahiri, The Ricci curvature of $\operatorname{Diff}\left(S^{1}\right) / \operatorname{SL}(2, R)$, Syracuse Univ. Prepr. SU-4238-377 (Feb. 1988).

3. M. Bowick and S. Rajeev, String theory as the Kähler geometry of loop space, Phys. Rev. Lett. 58 (1987), 535-538.

4. __ The holomorphic geometry of closed bosonic string, Nuclear Phys. B 293 (1987), 348384.

5. P. Griffiths, Periods of integrals on algebraic manifolds, Bull. Amer. Math. Soc. 75 (1970), 228-296.

6. _ Periods of integrals on algebraic manifolds III, Publ. Math. IHES, vol. 38, 1970.

7. D. Hong and S. Rajeev, Universal Teichmüller space and Diff $\left(S^{1}\right) / S^{1}$, Comm. Math. Phys. 135 (1991), 401-411.

8. Y. Katznelson, S. Nag, and D. Sullivan, On conformal welding homeomorphisms associated to Jordan curves, Ann. Acad. Sci. Fenn. Ser. A I Math. 15 (1990), 293-306.

9. S. Nag, Canonical measures on the moduli spaces of compact Riemann surfaces, Proc. Indian Acad. Sci. Math. Sci. 99 (1989), 103-111.

10. _ Non-perturbative string theory and the diffeomorphism group of the circle, Proc. Internat. Sympos. Topological and Geometrical Methods in Field Theory, Turku, Finland 1991 (J. Mickelsson, ed.), World Scientific (to appear).

11. The complex analytic theory of Teichmüller spaces, John Wiley, Interscience, New York, 1988.

12. S. Nag and A. Verjovsky, $\operatorname{Diff}\left(S^{1}\right)$ and the Teichmüller spaces, Comm. Math. Phys. 130 (1990), 123-138. In two Parts. Part I by S. N. and A. V.; Part II by S. N.

13. G. Segal, Unitary representations of some infinite dimensional groups, Comm. Math. Phys. 80 (1981), 301-342.

14. G. Segal and G. Wilson, Loop groups and equations of KdV type, Publ. Math. IHES, vol. 61, 1985.

15. C. L. Siegel, Symplectic geometry, Academic Press, New York, 1964.

16. E. Witten, Coadjoint orbits of the Virasoro group, Comm. Math. Phys. 114 (1981), 1-53.

17. B. Zumino, The geometry of the Virosoro group for physicists, Cargese 1987 (R. Gastmans, ed.), Plenum Press, New York.

The Institute of Mathematical Sciences, C.I.T. Campus, Madras 600 113, INDia

E-mail address: nag@imsc.ernet.in 